\title{
Ángel Anguiano, director del Observatorio Astronómico Nacional de Chapultepec y la construcción social en torno a la observación del tránsito de Venus por enfrente del disco solar en 1882
}

\author{
S. Zueck \\ Departamento de Geografía Social, Instituto de Geografía, \\ Universidad Nacional Autónoma de México.
}

Received 21 February 2020; accepted 8 September 2020

\begin{abstract}
La investigación examina la gestión del ingeniero civil y arquitecto Ángel Anguiano, director de la agencia estatal el Observatorio Astronómico Nacional de Chapultepec, quien durante el periodo presidencial del general Manuel González Flores, obtuvo considerables recursos económicos para modernizar el observatorio e insertarlo en la comunidad científica internacional. Mostramos que, a la par de haber sido contratado para contribuir en un mapa más preciso de la república mexicana, persuadió a la burocracia del presidente González para que patrocinaran su participación en la colaboración en torno a la observación del tránsito de Venus por enfrente del disco solar en el año de 1882 y calcular la distancia de la Tierra al Sol. Revelamos su habilidad para negociar con los funcionarios públicos la compra de modernos instrumentos ópticos, y su liderazgo para organizar delegaciones nacionales y diplomáticas a fin de albergar una comisión francesa. Las fuentes primarias de información las obtuvimos en documentos oficiales nacionales y franceses que evidencian el establecimiento de redes científicas capitalizadas rápidamente. Demostramos que la participación del Observatorio Astronómico Nacional se debió a que Ángel Anguiano ansiaba pertenecer al selecto círculo intelectual de la astronomía internacional e incursionar en las nuevas rutas de la astronomía propuestas por el grupo hegemónico francés, y no a una política científica de estado.
\end{abstract}

Descriptores: Ángel Anguiano; Observatorio Astronómico Nacional de Chapultepec; tránsito de Venus; Secretaría de Fomento; Colonización; Industria y Comercio.

The research examines the management of the civil engineer and architect Ángel Anguiano, director of the state agency the National Astronomical Observatory of Chapultepec, who during the presidential period of General Manuel González Flores, obtained considerable financial resources to modernize the observatory and insert it into the international scientific community. We show that, in addition to being hired to contribute to a more accurate map of the Mexican republic, he persuaded the bureaucracy of President González to sponsor his participation in the collaboration around the observation of the transit of Venus in front of the solar disk in the year 1882 and calculate the distance from the Earth to the Sun. We reveal his ability to negotiate with public officials the purchase of modern optical instruments, and his leadership to organize national delegations, and diplomatic, to host a French commission. The primary sources of information were obtained in national and French official documents evidencing the establishment of a rapidly capitalized scientific networks. We demonstrate that the participation of the National Astronomical Observatory in these networks were due to the desire of Ángel Anguiano to be a member of a select circle of international astronomers and intellectuals, and to venture into the new routes of astronomy proposed by the French hegemonic group, and not to a scientific state policy.

Keywords: Ángel Angiano; National Astronomical Observatory of Chapultepec; Transit of Venus; Ministry of Development, Colonization, Industry and Commerce.

DOI: https://doi.org/10.31349/RevMexFisE.18.143

\section{Introducción}

En México, observar el Sol es una tradición histórica, debido que diversas cosmovisiones prehispánicas lo identificaron como una de sus principales deidades, como por ejemplo Tonatiuh entre los mexicas y entre los mayas como Kin. Acorde a lo anterior, no es de sorprender que alguno de los tránsitos de Venus frente al disco solar fuera contemplado a simple vista por los expertos observadores mayas entre el siglo XIII y XIV. Actualmente resulta difícil imaginar que pudieran hacerlo sin instrumentos, pero existe evidencia de que es visible si interfiere una ligera nubosidad y que coincida con el amanecer o el anochecer o inclusive a través de un método de proyección [1].

Prueba de ello, son las pinturas datadas con técnicas de radiocarbono de entre el año 1200 y 1350 a.n.e., que se en- cuentran plasmadas en un muro de un edificio ubicado en la plaza central de la ciudad de Mayapán en el estado de Yucatán, México. En la construcción, orientada respecto a la trayectoria del planeta Venus, se encuentra la llamada Sala de los Frescos, en donde se aprecian unos personajes que representan a este planeta, y que van descendiendo por el interior de imágenes del Sol, por lo que se infiere sean un registro del tránsito del año de 1275 [2].

Dando un salto en el tiempo, y aunque se reconoce la contribución de los neerlandeses Zacharias Jansen (1583-1638) y de Hans Lippershey (1570-1619) a la invención del telescopio, nosotros partimos de la fecha histórica en que el astrónomo Galileo Galilei (Pisa, 1564-1642) publicó su obra Sidereus Nuncius en el año de 1610. Se debe a que en ella dio cuenta de un nuevo telescopio de tipo refractor que le permitió observar con mejor definición objetos lejanos al planeta 
Tierra [3]. Con el tiempo, gracias a su curiosidad científica y al avance tecnológico del telescopio, realizó importantes hallazgos sobre la luna, las nebulosas, los satélites de Júpiter y las manchas solares. Desde entonces, el peculiar paso de Venus por enfrente del disco solar se ha presentado hasta hoy en ocho ocasiones, siendo los más recientes los del 8 de junio de 2004 y el del 5 de junio de 2012. Esto se debe a que sigue un patrón que se repite cada 243 años. Dentro de este periodo ocurren cuatro pares de tránsitos con intervalos entre ellos, es decir, transcurridos 105.5 años ocurrirá uno, otro en un lapso de 8 años, el siguiente en 121.5 y nuevamente 8 años después hasta completar el patrón.

Esto se debe al tiempo que tarda cada uno de los dos planetas en completar una vuelta alrededor del Sol y a el ángulo que existe entre las órbitas de los dos planetas. Es decir, cada vez que la Tierra completa 8 vueltas alrededor del Sol, Venus lo completa en 13 y aunque en este tiempo Venus pasa 5 veces delante de la Tierra no se presenta el tránsito. Esto se debe a que la órbita de Venus está inclinada con respecto a la órbita de nuestro planeta por un ángulo de $4.3^{\circ}$ por lo que la mayoría de las veces que Venus pasa delante de la Tierra lo vemos ligeramente por encima o por debajo del Sol [4].

Como mostramos en la Fig. 1, la alineación del Sol, Venus y Tierra debe coincidir con la línea (imaginaria) que une al Sol con los nodos de las órbitas de los dos planetas. Esta situación nos permite observar a Venus desde nuestro planeta, como un punto negro que lentamente atraviesa el disco del Sol en dirección este a oeste.

Ubicados en el siglo XVIII, el astrónomo inglés Edmund Halley (Londres, Reino Unido, 1656-1742), a pesar de que la vida le impediría observar los tránsitos de 1761 y 1769, fue el primero en plantear en el año de 1716 que la observación del tránsito podría ser utilizada para medir la paralaje solar [5]. Halley, con base en la tercera ley de Johannes Kepler (Würtemburg, actual Alemania, 1571-1630), propuso observar el paso de Venus frente al disco solar desde dos latitudes para determinar con precisión la distancia Tierra-Venus y por lo tanto conocer el valor de la Unidad Astronómica. Solo para dejar claras las ideas generales del proceso, presentamos el escenario más simple de la propuesta de Halley.

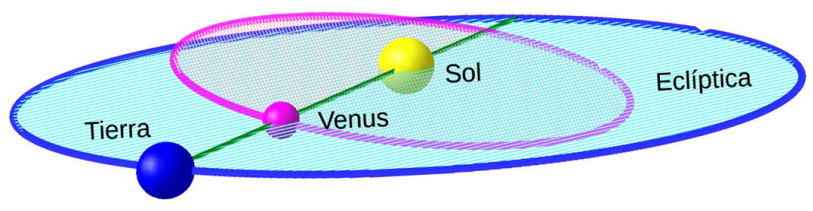

Figura 1. Diagrama esquemático de la alineación de la Tierra,Venus y el Sol durante el tránsito, la órbita de la Tierra (marcada en azul) define el plano de la eclíptica y la órbita de Venus (marcada en magenta) que tiene una inclinación de 3.4 grados con respecto al plano de la eclíptica.

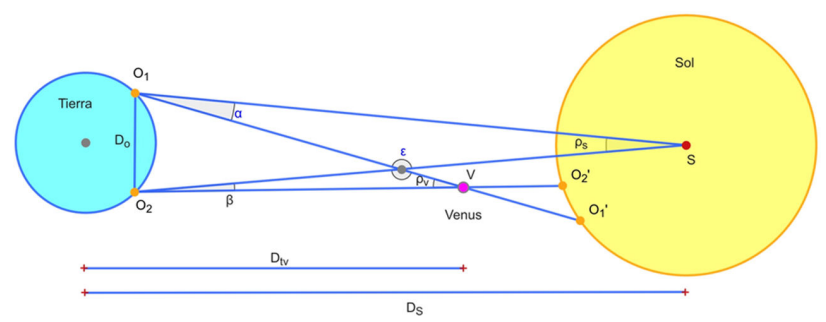

FIgURA 2. Esquema simplificado de la propuesta de Halley para determinar la distancia Sol-Tierra $\left(D_{s}\right)$ en función de la distancia $D_{o}$ entre dos observadores $\left(O_{1}\right.$ y $\left.O_{2}\right)$ que miden los ángulos de paralaje $\alpha$ y $\beta$ de Venus durante su paso por el disco solar.

En la Fig. 2 se pueden notar los observadores posicionados en los puntos $O_{1}$ y $O_{2}$ que están situados en dos latitudes diferentes de la Tierra (y separados por la distancia $D_{o}$ ) pero en la misma longitud para simplificar el desarrollo. Para estos observadores la trayectoria de Venus sobre el disco solar pasa sobre los puntos marcados como $O 1^{\prime}$ y $O 2^{\prime}$. Como se ve en la figura se forman dos triángulos que comparten el vértice central y por lo tanto tienen al mismo ángulo $\epsilon$. Del triángulo superior se tiene que $\alpha+\epsilon+\rho_{s}=180^{\circ}$, mientras que del triángulo inferior se tiene $\beta+\epsilon+\rho_{v}=180^{\circ}$, en donde $\rho_{s} \mathrm{y}$ $\rho_{v}$ son los paralajes del Sol y Venus, respectivamente.

Por otra parte, tomando en cuenta la definición de paralaje se tiene que $\rho_{v}=D_{o} / D_{t v}$ y $\rho_{s}=D_{o} / D_{t}$ en donde $D_{t v}$ y $D_{t}$ son la distancia Tierra-Venus y Tierra-Sol, respectivamente, por lo que $\alpha-\beta=\rho_{v}-\rho_{s}=D_{o} /\left(D_{t v}-D_{T}\right)$ y sustituyendo el valor relativo de la distancia Tierra-Venus dado por la tercera ley de Kepler, tenemos que $\alpha-\beta=D_{o} /\left(0.72 D_{T}\right)$, lo que significa que si se conoce con precisión $\alpha-\beta$, esto es la diferencia de ángulos medidos por cada observador con respecto del centro del disco solar, podemos deducir la distancia Tierra-Sol. Desafortunadamente $\alpha-\beta$ es muy pequeña para medirla directamente, pero se puede inferir usando la longitud de las cuerdas que traza el paso de Venus sobre el disco solar. Para lograrlo, se mide el tiempo de inicio y final de dicho paso en cada uno de los puntos de observación.

Lo que en realidad se mide son los tiempos de los llamados cuatro contactos, es decir, cuando a la entrada o inicio del evento Venus toca por primera y última vez el limbo solar, y los dos contactos finales se dan de forma similar, pero cuando éste sale del disco solar.

En la Fig. 3 observamos que suponiendo que la velocidad de Venus es constante para ambos observadores, la distancia $\alpha-\beta$ se puede escribir en función de la diferencia de tiempos tomando en cuenta los triángulos $O_{1}^{\prime} S D$ y $\mathrm{O}_{2}^{\prime} S B$, en donde las cuerdas $C D$ y $A B$ corresponden a los tiempos del tránsito medidos por $O_{1}$ y $O_{2}$, es decir $T_{1}$ y $T_{2}$, respectivamente. Dado que el ángulo $\delta$ es muy pequeño, podemos suponer que $\cos (\delta)=1 \mathrm{y}$ las diferencias de los catetos de los triángulos son: $\alpha-\beta=R \sin (\theta) \sin (\delta)$ y $T_{1}-T_{2}=R \cos (\theta) \sin (\delta)$, la diferencia de tiempo relativa es $\Delta T_{r}=\left(T_{1}-T_{2}\right) / T_{1}=(\cos (\theta) \sin (\delta)) / \sin (\theta)$, de donde se despeja $\sin (\delta)$ para obtener $\alpha-\beta=R \Delta T_{r} \sin ^{2}(\theta) / \cos (\theta)$, Finalmente, recordando que $\alpha-\beta=D_{o} /\left(0.72 D_{T}\right)$ se tiene que $D_{o} /\left(0.72 D_{T}\right)=R \Delta T_{r} \sin ^{2}(\theta) / \cos (\theta)$, esto es, se ob- 


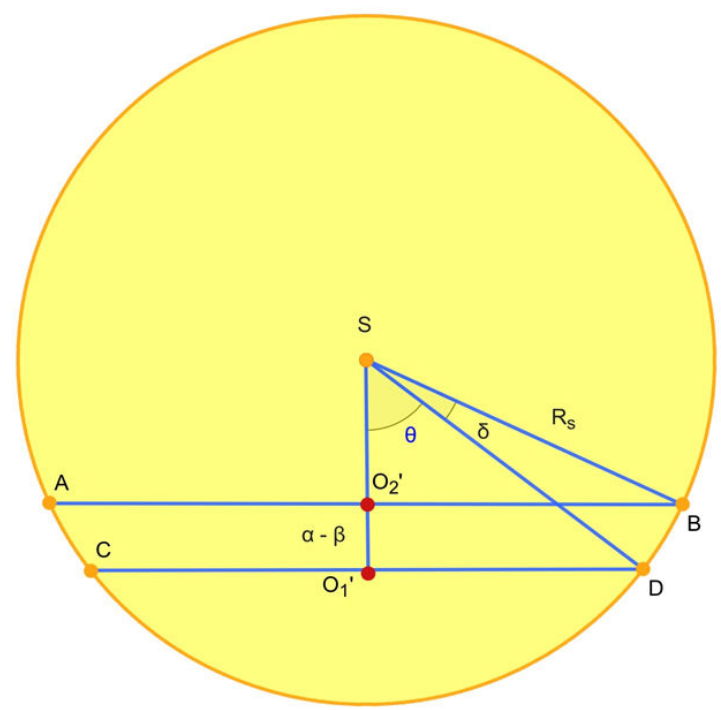

FIgURA 3. Trayectorias de Venus $(A B$ y $C D)$ observadas por $O_{1}$ y $\mathrm{O}_{2}$ durante el paso de Venus por el disco solar. $R s$ es el radio solar que corresponde a 16 min de arco.

tiene la distancia Tierra-Sol a partir de parámetros observados. Con todo, observar el eclipse no fue cosa fácil debido a un fenómeno óptico que llamaron "ligamento negro" o "gota negra" y que aparece cuando hace contacto con el limbo solar impidiendo computar el tiempo con exactitud. Actualmente sabemos que se debe es una combinación de factores tales como la calidad del cielo del lugar donde se hacen las observaciones, la óptica del telescopio y lo más importante, el oscurecimiento del limbo del disco solar [6]. En síntesis, en la observación del tránsito entran en juego los instrumentos, las habilidades del observador, el ligamento negro y la incertidumbre de las condiciones meteorológicas.

Evidentemente este suceso astronómico no se presenta muy seguido, razón por el cual la globalizada comunidad astronómica que nos interesa investigar, no quiso dejar pasar la oportunidad de aventurarse a cronometrar el paso de Venus por enfrente del disco solar.

En este orden de ideas, nosotros proponemos la participación de la agencia mexicana, el Observatorio Astronómico Nacional de Chapultepec (de aquí en adelante OANCH) en la observación internacional del tránsito de Venus por enfrente del Sol realizada en 1882, resaltando la figura del primer director, el ingeniero civil y arquitecto José Ángel Anguiano Limón (Encarnación de Díaz, Jalisco, 1840-1921), contratado por el Estado mexicano para contribuir en la resolución de una nueva carta geográfica de la república mexicana. El cargo lo desempeñó por 23 años hasta que fue nombrado director de la Comisión Geodésica Mexicana.

Ángel Anguiano tuvo la opción de observar el fenómeno natural con los instrumentos ópticos del OANCH o de ignorarlo, entonces nos preguntamos ¿porqué decidió colaborar en la organización internacional que buscó la resolución de un problema científico de frontera?

Planteamos que el director anheló incursionar en las prácticas institucionales formales del grupo dominante de la astronomía internacional. Para alcanzar su objetivo, aprovechó sus nexos con los burócratas a quienes el presidente de la república mexicana, el general Manuel González Flores (Matamoros, México, 1833-1893) dio empleo. A través de ellos obtuvo los recursos económicos para 1) viajar a Europa a negociar la compra de telescopios con las principales casas constructoras de instrumentos ópticos y 2) establecer redes científicas con los directores de los observatorios europeos.

Mientras que desde el punto de vista de la historia de la ciencia se considera a nivel internacional a Ángel Anguiano como un astrónomo más [7], nosotros presentamos un enfoque sociológico distinto; nos apoyamos en el concepto de hegemonía cultural propuesto por el sociólogo Antonio Gramsci (1891-1937) quien afirmó que ésta es una imposición pacífica de ideas y valores del mundo por parte de un grupo dominante. Explicó que sus integrantes generalmente pertenecen a la clase gobernante y para lograr que sus postulados se han aceptados por la sociedad de una manera pasiva, se recurre a los llamados "intelectuales orgánicos" llamados así debido a que forman parte de la burocracia y/o están ligados a la ideología hegemónica [8].

Con base en lo anterior, constatamos que el Estado mexicano, a través de la Secretaría de Fomento, Colonización, Industria y Comercio apoyó al director del OANCH para que adquiriera instrumentos ópticos como los de los observatorios líderes de la astronomía y de paso, obtuviera de primera mano la información científica oficial para realizar la observación del tránsito de 1882. Destacamos que, en la consecución de sus metas, sorteó desde problemas políticos hasta logísticos y mostramos su empeño y capacidad intelectual, por lo que para el año de 1883 ya formaba parte de la globalizada élite astronómica de finales del siglo XIX.

Los límites cronológicos de la investigación inician con la fundación del OANCH en 1876 y concluyen cuando los resultados de la última observación del siglo XIX se publicaron en la prensa nacional en 1883. Abarca un lapso de la primera etapa del porfiriato, durante el cual se llevaron a cabo importantes acciones en la educación superior como la Ley de instrucción para la Escuela Nacional de Ingenieros y de Agricultura, y científicas, al impulsar la creación de instituciones abocadas al reconocimiento nacional de carácter científico como el Observatorio Astronómico Nacional y la Comisión Geográfica Exploradora [9].

Precisamos que en este tiempo fue elegido como presidente de la república mexicana el general Porfirio Díaz (Oaxaca de Juárez, México, 1830-París, Francia, 1915) para el periodo de 1876 a 1880, seguido por el del general Manuel González Flores (1880-1884), quienes compartieron la visión positivista de que la ciencia llevaría a México al progreso .

El estudio se engarza con la importante Secretaría de Fomento, Colonización, Industria y Comercio (de aquí en adelante Secretaría de Fomento) y en términos generales su objetivo fue el estudio y reconocimiento del territorio nacional. Debido a su orientación práctica y utilitarista, para el último tercio del siglo XIX fue la institución del estado más impor- 
tante y con mayor presupuesto [10]. Durante el periodo que nos ocupa, la Secretaría de Fomento fue presidida por importantes liberales que tuvieron una participación activa en la organización de las políticas públicas de la nación, como el literato Vicente Riva Palacio (28 de noviembre de 1876 30 de noviembre de 1880) seguido por el breve periodo del mismo general Porfirio Díaz (1 de diciembre de 1880 - 27 de junio de 1881), y finalmente por el del chihuahuense, general Carlos Andrés Pacheco Villalobos (27 de junio de 1881 - 8 de enero de 1892).

Los documentos base de este trabajo son los informes publicados en las Memorias de la Secretaría de Fomento, Colonización, Industria y Comercio y los editados para el Anuario del Observatorio Astronómico Nacional de Chapultepec-posteriormente de Tacubaya-; las fuentes secundarias las encontramos en los periódicos de la época como El Monitor Republicano y el Siglo XIX. Debido al carácter internacional del tema, recurrimos a fuentes documentales francesas tales como la revista Comptes Rendus des Seances de l'Academie des sciences y la Memories de l'Academie des sciences de l'Institut de France.

En razón a lo expuesto, iniciamos con un breve contexto histórico que se centra en la designación del primer director del OANCH y sus gestiones para negociar recursos económicos. En la segunda sección discernimos sobre la organización social internacional en torno a la observación del 6 de diciembre 1882. En un tercer momento, disertamos sobre la táctica para asegurar la compra de instrumentos y la estrategia mediática para que participaran las delegaciones mexicana y francesa. Continuamos con la identificación de los expertos que participaron en la observación en la república mexicana y sus resultados. Finalmente, presentamos las conclusiones.

\section{Las redes científicas}

Al triunfo de la revolución de Tuxtepec en 1876, cuyo objetivo fue impedir que el presidente Lerdo de Tejada se reeligiera, provocó que la estructura burocrática del aparato gubernamental de la república mexicana cambiara. En un nuevo orden social quedaron desempleados los que habían participado en la administración de Tejada, así que el jalisciense José Ángel Anguiano Limón, que al terminar sus estudios de ingeniero civil y arquitecto había sido contratado como inspector de caminos de Michoacán (1868-1870), pensó que aprovechando su experiencia como profesor de matemáticas en la Universidad de San Nicolás de Hidalgo, lo mejor sería poner una escuela particular [11].

Con todo, el presidente interino Juan Nepomuceno Méndez, con el apoyo del Secretario de Fomento, Vicente Riva Palacio y el de Justicia e Instrucción Pública, el abogado Ignacio M. Altamirano, lo designaron en el año de 1876 como director del Observatorio Astronómico Nacional; además había que dirigir uno meteorológico en donde se efectuaría la recolección metódica de datos que servirían como base del pronóstico del tiempo y uno para medir las variaciones del campo geomagnético.
Como ya mencionamos, el objetivo particular del observatorio fue contribuir a la carta exacta de México, para lo cual era necesario habilitar un edificio idóneo. El sitio ideal fue una fortificación que se empezó a construir en el año de 1785 en lo alto de un cerro llamado Chapultepec rodeado por un bosque y un lago. Su ubicación a 2 mil 325 metros de altura sobre el nivel del mar permitió una excelente observación de la bóveda celeste y una importante avenida llamada Paseo de la Reforma, facilitó el traslado de los astrónomos a la ciudad de México en donde estaban ubicadas las oficinas de la Secretaría de Fomento, contigua a la Escuela Nacional de Ingenieros (de aquí en adelante ENI) y al Observatorio Astronómico Central (de aquí en adelante OAC), localizado en la azotea del Palacio Nacional.

Un breve recuento del sitio conocido como el Palacio de Chapultepec, alude al general Pedro García Conde (Arizpe, Sonora, 1806-1851) quien intentó instalar un observatorio astronómico ahí, pero debido a la guerra de 1847 con los estadounidenses lo tuvo que abandonar. El segundo intento corrió con la misma suerte, ya que durante la intervención francesa (1862-1867) el ingeniero Francisco Díaz Covarrubias (Xalapa, Veracruz, México, 1833-1889) tuvo que desistir de la misma idea; el recinto también se utilizó como escuela militar y residencia imperial, y después de las invasiones militares extranjeras ya mencionadas, se encontraba en un total abandono. En este ambiente, Riva Palacio encomendó la reconstrucción del sitio a los ingenieros civiles y arquitectos egresados de la Academia de San Carlos, Juan N. Anza y el mismo Anguiano. Como arquitecto, se encargó de la restauración del observatorio y del remozamiento del bosque con un presupuesto de $\$ 21,597.10$ para el año fiscal de 1877 y otro tanto para el de 1878 [12].

Estratégicamente la inauguración oficial tuvo lugar el cinco de mayo de 1878 y contó con la presencia del presidente Porfirio Díaz, que festejó ahí la victoria de las fuerzas armadas mexicanas en contra del poderoso ejército francés en el año de 1862. Cabe señalar que una de las cinco batallas que tuvieron lugar en el conocido Fuerte de Loreto y Guadalupe, cercano a la ciudad de Puebla, la ganó Díaz .

Utilizando los modernos instrumentos como el telégrafo y el teléfono, se hizo una demostración del cálculo de la posición geográfica del OANCH y de la distancia entre éste y el OAC aplicando el método de intercambio de señales telegráficas. El Central, fue una agencia estatal a cargo del experto en métodos astronómicos, el ingeniero Francisco Jiménez, que también fue recontratado a pesar de haber apoyado a los regímenes anteriores. Casualmente, el tránsito de Mercurio por enfrente del Sol fue visible en la Ciudad de México al día siguiente, y a manera de ensayo, aplicaron el método de triangulación.

De acuerdo al contrato laboral con la Secretaría de Fomento, Ángel Anguiano realizó una multiplicidad de tareas gracias a la experiencia de campo adquirida como inspector general de caminos del país (1870-1876). Algunas de estas fueron la determinación de la longitud de las principales capitales de la república mexicana con respecto al observatorio, 
así como el establecimiento de los límites de los estados de Guanajuato y Jalisco y de Guatemala con México. Además coordinó el registro exacto del tiempo y los datos sobre el aspecto general del cielo, mismos que fueron publicados en la sección astronómica del Boletín del Ministerio de Fomento de la República Mexicana periódico de divulgación patrocinado por Fomento [13].

A título personal gestionó, preparó y editó el libro de difusión el Anuario del Observatorio Astronómico Nacional de Chapultepec que también fue patrocinado por la Secretaría. Por cierto, en los contenidos de los cuatro primeros números (1880 a 1883) compartió con los lectores -integrantes de la élite intelectual científica mexicana- la información relacionada con la observación de diciembre de 1882 [14]. Además, comenzó a dar clases como profesor sustituto del ingeniero Leandro Fernández (Nombre de Dios, Durango, México, 1851-1921) que impartía la materia de geodesia y astronomía práctica en la ENI.

Ante el apremio de tener que realizar muchas tareas, Anguiano manifestó respetuosamente a las autoridades que era “... indispensable aumentar desde luego el personal del observatorio, pues muchas veces la buena voluntad y toda la dedicación posible no bastan para alcanzar un fin deseado" [15].

En esta especificidad histórica, pero en otro espacio geográfico, el almirante Ernest Mouchez (Madrid, España, 18211892), director del Observatoire de Paris, convocó a la comunidad internacional a una serie de reuniones científicas relacionadas con el futuro tránsito de 1882 del 5 al 13 de octubre de 1881 [16]. A pesar de las diferencias lingüísticas, culturales o ideológicas y aunque los separaban grandes distancias -en algunos casos transoceánicas- todos tenían un objetivo científico común: calcular la distancia del planeta Tierra al Sol. Indiscutiblemente para la comunidad astronómica sería la última oportunidad en sus vidas de participar en el más importante evento astronómico del siglo XIX, ya que el siguiente par de alineamientos se presentarían en el siglo XXI.

Sabiendo que en esta ocasión sería visible en toda la república mexicana, Anguiano cabildeó a las nuevas autoridades del gabinete empleado por el presidente Manuel González. Ayudó que el ingeniero Manuel Fernández Leal (Xalapa,Veracruz, 1831-1909), al regresar de la observación del tránsito de 1874 en Asia, se incorporara dentro de la burocracia y en ese momento ocupara interinamente la cartera de Fomento [17]. Teniendo intereses en común y queriendo ser visibles en la comunidad internacional, éste le autorizó un viaje a Europa, para que se contactara con las principales fábricas de instrumentos ópticos de su elección; además debía visitar los observatorios del viejo continente para que averiguara sobre los preparativos para observar el paso de Venus por enfrente del disco del Sol de 1882.

Para llevar a cabo la misión de seis meses de duración, el secretario de Hacienda Landero y Cos proveyó 300 pesos para viáticos y mil 800 de salario. Aprovechando los modernos medios de comunicación, el 27 de mayo de 1881 Anguiano partió en el nuevo Ferrocarril Mexicano al puerto de Veracruz para embarcarse rumbo al de Nueva York, desde donde se dirigió al de Liverpool en el trasatlántico City of Richmond [18].

\section{Los anfitriones}

En esta globalizada comunidad científica relacionada con la astronomía, todos aquellos que contaron con el apoyo económico de sus gobiernos tuvieron como hábito acudir a otros centros astronómicos para tomar nota de sus prácticas científicas; un ejemplo de ello es Federico Augusto Oom, futuro director del Observatorio Astronómico de Lisboa, que se entrenó como astrónomo en el Observatorio de Pulkovo, en San Petersburgo, Rusia, del año 1858 a 1863 [19].

En el caso que nos ocupa, la prioridad de la travesía indudablemente fue comprar exclusivos instrumentos ópticos a las casas constructoras que monopolizaban el mercado científico astronómico europeo. Acorde a lo anterior, una de las primeras acciones del director del OANCH fue reunirse en Londres con el dueño de la compañía Troughton \& Simms y a quien el observatorio y las comisiones geográficas del territorio nacional ya le habían comprado algunos instrumentos. Simms y sus colegas, se comprometieron a entregar un círculo meridiano y recomendaron al reconocido constructor de grandes telescopios, el irlandés Howard Grubb, establecido en la ciudad de Dublin. Este sugirió la construcción de un telescopio con montura ecuatorial con un objetivo de 15 pulgadas de diámetro en el objetivo y de seis yardas de distancia focal que debido a su tamaño fue conocido como "el gran ecuatorial". Encontramos que en el informe de actividades que Anguiano envió al nuevo Secretario de Fomento, el general Carlos Pacheco, mencionó que con tal de que el instrumento llegara a Chapultepec a tiempo ofreció al constructor una remuneración extra por el artefacto, aunque como medida precautoria, ordenó otro de 6 pulgadas de abertura; por cierto que Grubb, receloso sobre la liquidez de su nuevo cliente estableció las condiciones de compra-venta [20]. Una vez estipulados los montos económicos finales y las bases contractuales con ambas compañías, el director del observatorio de Chapultepec recibió la autorización para cerrar el negocio hasta el 22 de octubre de 1881.

En esta situación, en el interín se dedicó a visitar observatorios europeos e indagar desde sus prácticas científicas y administrativas hasta sobre sus bibliotecas y arquitectura.

Un breve resumen del recorrido nos muestra sus excelentes habilidades como gestor de redes, ya que en el Reino Unido tomó ventaja del encuentro con Simms, que le presentó al astrónomo William Chistie, subdirector del Royal Observatory of Greenwich; éste le mostró, entre muchos otros artefactos, los instrumentos para medir el tiempo con precisión que habían comprado a la compañía Troughton y Simms, mientras que Grubb lo enlazó con el Dunsink Observatory.

En Europa continental visitó desde el prestigioso observatorio del Collegio Romano, especializado en astronomíafísica, el modesto Observatorio Astronómico Capodimonte 
de Nápoles, el pequeño Observatoire de Genève, hasta el ostentoso Universitäts-Sternwarte Wien.

Aprovechando sus conexiones con la cancillería mexicana en Europa, logró ser recibido en el Observatoire Royal de Belgique gracias al ministro de México en Bruselas, el diplomático Ángel Núñez Ortega; al Observatorio de marina de San Fernando llegó acompañado del cónsul de México en Cádiz, el señor Agustín Sánchez Antuñano; Jésus Zenil, secretario de la legión mexicana en Madrid lo condujo al Observatorio Astronómico de Madrid y Andrés Aldasoro al Berliner Sternwarte [21]. De toda la gira científica destaca la que realizó en compañía de su amigo el astrónomo Francisco Díaz Covarrubias a uno de los observatorios más antiguos de Europa, el Observatoire de Paris (1667) encabezado por el almirante Ernest Mouchez; al meteorológico de Montsouris (1876) al servicio de la marina francesa y en donde se estableció transitoriamente uno de los meridianos de referencia de la época y al de Meudon (1876), especializado en astronomíafísica y dirigido por el experto Jules Janssen; de acuerdo con Anguiano, en conjunto denotaban un profundo símbolo de orgullo nacional debido a sus importantes aportes a la astronomía internacional. En ese sentido, fue fundamental la visita que realizó a las sedes francesas ya que adquirió de primera mano la información metodológica y técnica que los astrónomos galos planeaban utilizar.

Este interés no nos debe sorprender ya que al igual que otros países latinoamericanos, en la república mexicana el paradigma cultural y científico fue el francés y la astronomía no fue la excepción; ejemplo de ello son los libros de texto (escritos en la lengua francesa) cuyos autores eran líderes de la ciencia y que fueron utilizados en la ENI para formar a los futuros ingenieros.

Mientras tanto, todos aquellos que interesados en el dilema del cálculo de lo que actualmente llamamos Unidad Astronómica se dieron cita en la Conference internationale du passage de Vénus que tuvo lugar del 5 al 13 de octubre de 1881en la ciudad de París. Destacamos el resultado de las cinco sesiones, en las cuales los representantes de los observatorios de Alemania, Reino Unido, Austria, Brasil, Chile, Dinamarca, España, Italia, Países Bajos, Portugal y Argentina, se pusieron de acuerdo en recomendar el método observacional y en los instrumentos ópticos.

Notamos que Anguiano no asistió a las reuniones pero que su observatorio aparece en la lista de instituciones científicas interesadas en la colaboración. La circunstancia nos hace pensar que Chapultepec fue considerado debido a que en la visita a las sedes parisinas se planteó que se estaba negociando la compra de instrumentos para observar el evento astronómico del 6 de diciembre de 1882 [22].

En este contexto, al igual que en el tránsito de 1874, los observatorios ubicados en el hemisferio donde no sería visible el fenómeno natural indicaron los sitios donde establecerían sus delegaciones foráneas. Advirtiendo que los franceses enviarían una de sus siete comisiones al Golfo de México, (en territorio de los Estados Unidos) en otra circunstancia social, Anguiano nuevamente aprovechó canales informales y en esta ocasión recurrió a su amigo, el diplomático jalisciense Leonardo López Portillo [23]. El objetivo fue concertar una reunión con Ernest Mouchez -independiente de los protocolos de las conferencias- que to y alentar al director galo de instalar un observatorio en la república mexicana. Con tal de capitalizar las mejores condiciones de observación, Mouchez ignoró los eventos bélicos pasados entre México y Francia, así como el sentimiento antifrancés que aún existía y como veremos más adelante, tomó en cuenta la sugerencia de Anguiano.

Hacemos notar que el estado otorgó un importante apoyo económico durante el viaje que duró el recorrido europeo, pero en ausencia de nuestro protagonista, el palacio de Chapultepec fue reclamado por el ejército. En consecuencia, supo que después de la observación tendría que: 1) trasladar los instrumentos ópticos -que todavía no adquiría- al edificio que ocupó la Escuela Militar ubicada en el edificio del antiguo arzobispado de la Villa de Tacubaya; 2) negociar con las autoridades de Fomento los fondos para construir un moderno observatorio similar a los de sus colegas europeos y con base en un proyecto ideado por él mismo.

\section{La estrategia de comunicación}

A su regreso del viejo continente en enero de 1882 y tomando en cuenta la premura del tiempo, una de las primeras acciones del director del OANCH fue escribirle al señor Eduardo De Santos con domicilio en la 46 Rué de Provence en París. De Santos fue el dueño de la compañía que fungió como casa de cambio y como gestora intermediaria entre el estado mexicano y los propietarios de las constructoras de instrumentos ópticos. Acorde a lo anterior, logró que se le enviaran 6 partidas mensuales de 5000 mil pesos mexicanos que se debieron cambiar a libras o francos, situación que disminuyó el valor nominal de la moneda original. Para darnos una idea de lo costoso que fue adquirir el equipo, durante este periodo el salario mínimo promedio de los habitantes de la república mexicana fue de 20 centavos.

Adicionalmente, De Santos medió con la casa Troughton y Simms la compra del fotoheliógrafo de Dallen-Meyer de cuatro pulgadas de abertura y cinco pies de distancia focal, y que también adquirieron las expediciones británicas, australianas y la de la India, además de cronómetros y péndu$\operatorname{los}$ [24].

Mientras tanto, en el observatorio de Chapultepec se contrató al ingeniero civil Emilio Baudouin (de nacionalidad mexicana) para proceder con la construcción de las planchas de cemento en donde se instalarían las cúpulas giratorias irlandesas de los telescopios. A la par, solicitó a su paisano jalisciense y bibliotecólogo del observatorio, el señor Apolonio Romo, tradujera al español las recomendaciones para cronometrar los contactos contenidas en las actas finales de la conferencia y publicadas en el cuaderno de le Ministère de l'Instruction Publique et des Beaux-Arts de France [25]. Un hecho aún más importante fue que las indicaciones establecidas con base en los valores de grupo de los astrónomos que 
asistieron a la conferencia de París se socializaron a través de los primeros cuatro números del Anuario del Observatorio Astronómico Nacional de Chapultepec, ya que fue una lectura obligada para los mexicanos interesados en la observación.

Por otro lado, por órdenes de Mouchez, el ingeniero hidrógrafo Anatole Bouquet de la Grye (Thiers, Francia, 1827-1909) solicitó consejo a Anguiano sobre el mejor sitio para instalar un campamento. Después de analizar los datos meteorológicos y dudar entre los estados de México o Puebla, Anguiano lo convenció de que sería una mejor idea ubicarse en el Fuerte de Loreto y Guadalupe, sitio en donde ya mencionamos, 20 años atrás se obtuvo una victoria en contra del poderoso ejército francés. Nuevamente, gracias a sus habilidades para cabildear con las autoridades mexicanas, el director del observatorio tramitó que les instalaran una línea de telégrafo desde el OANCH al Fuerte, la construcción de un camino y una escolta para su seguridad [26].

Sin embargo, y a pesar de que contó con el apoyo económico del estado para equiparlo al igual que los mejores del mundo, contradictoriamente no se le otorgaron plazas, por lo que hubo que optimizar el rendimiento de los escasos trabajadores. Un ejemplo es el del bibliotecario/meteorólogo cuyas competencias científicas para ser contador de cronómetros las adquirió cuando Anguiano, como director general de caminos, lo adiestró participando en algunas de las comisiones del reconocimiento del país.

\section{La masa crítica: los observadores}

Esta sección la iniciamos formulando la siguiente pregunta: dentro de la república mexicana ¿quiénes podían tener los conocimientos astronómicos para participar en la observación del tránsito de Venus organizada por Anguiano?

En una atmósfera cultural en donde la masa global de los nueve millones de habitantes de México hablaba otra lengua y no sabía leer, fue la ENI el sitio en donde una élite intelectual tenía acceso a la transferencia del conocimiento que los integrantes del observatorio dominaban. No nos debe sorprender que Anguiano enviara una carta de invitación y un ejemplar del Anuario para el año de 1883 al director de la ENI, el ingeniero en minas Antonio del Castillo Patiño (Huetamo, Michoacan, 1820-1895). En respuesta, la escuela organizó un grupo liderado por el ingeniero topógrafo Francisco Rodríguez Rey, que en ese momento trabajaba como conservador de gabinetes de topografía, geodesia y astronomía y que además era empleado del OANCH. El equipo de observadores estuvo integrado por los alumnos que ya habían acreditado el curso de geodesia y astronomía práctica, entre quienes se encontraban Pedro Gutiérrez, Carlos Sellerier, Ezequiel Pérez, José Tamborrel y Juan de Dios Fleury [27]. La escuela en realidad sólo se abocó a arreglar la cúpula giratoria y proporcionó algunos de los mismos instrumentos antiguos y usados que habían viajado con la comisión científica mexicana que observó el tránsito de 1874 en el Imperio del Japón. Otro grupo comprometido a participar en el evento fue el del nuevo director del OAC, el ingeniero Leandro Fernández que sustituyó a Jiménez cuando falleció en 1881. Como prueba de la escasez de instrumentos, Fernández usó un telescopio cenital que fue utilizado por Díaz Covarrubias en 1874. Desde la azotea de la casa del general Vicente Riva Palacio, el aficionado a la astronomía Juan Nepomuceno Adorno, a través de un instrumento de su invención, observó la imagen del Sol y del planeta reflejada en un vidrio.

En contraste, el para entonces nuevo Observatorio Astronómico Nacional de Chapultepec fue la única institución equipada con modernos instrumentos ópticos de primer orden y contradictoriamente carecía de trabajadores suficientes para hacerlos funcionar. Recordemos que el cometido de los trabajadores de la institución fue rectificar desde las coordenadas geográficas de las más importantes ciudades de los estados de la república mexicana, hasta las de la nueva frontera entre los Estados Unidos, por lo que se trasladaban a donde eran requeridos. Por si fuera poco, los futuros ingenieros geógrafos aún se estaban formando en las aulas de la ENI. Para solucionar el problema, el director no tuvo otra alternativa y recurrió temporalmente al personal de la misma Escuela Militar o del Estado Mayor y se abocó a entrenarlos como observadores.

Cuatro años después de la inauguración, el observatorio, estaba organizado en cuatro departamentos y contaba con un personal disciplinado y entrenado para colaborar en la gran cooperación científica internacional. En la sala llamada del ecuatorial, Apolonio Romo utilizó el cronómetro y José López Acevedo fue responsable de las observaciones meteorológicas. En la sala destinada al instrumento altazimutal, Felipe Valle y Carmona tuvo la responsabilidad de cronometrar los cuatro contactos con el auxilio de Francisco Girón, encargado de la comunicación telegráfica, cronográfica y telefónica. En la sala fotográfica el mayor de caballería de la Secretaría de Guerra, el señor Teodoro Quintana y su ayudante Juan Quintas Arroyo, manejaron el fotoheliógrafo; por otro lado, los integrantes del Estado Mayor, Antonio R. Flores y Enrique Olivares observaron en la sala meridiana utilizando un telescopio de pasos Ertel [28].

El director Anguiano manejó el telescopio de 6 pulgadas debido a que el refractor grande no llegó. De lo que no cabe duda es que después de que la Secretaría de Fomento aprobó pagar otra cuota extra al constructor, el oneroso instrumento llegó a México en 1883 y se instaló provisionalmente en el patio del ex arzobispado, mientras Anguiano construía el nuevo Observatorio Astronómico Nacional de Tacubaya .

\subsection{Expediciones en otros territorios de la república mexicana}

Al igual que los observatorios extranjeros que ubicaron delegaciones en varios sitios del continente en donde sería visible el tránsito, Anguiano recurrió a algunos integrantes de las comisiones del reconocimiento nacional que se encontraban en diferentes sitios del país. Esta élite formada en las mejores 
instituciones de educación superior del país fue contratada por la sección de cartografía de la Secretaría de Fomento o por la Comisión Geográfica Exploradora, quienes utilizaban antiguos instrumentos astronómicos deteriorados por el uso.

Iniciamos con los señores Gabriel Castaños y Carlos F. Landero, jefe de la Comisión Científica Exploradora del Pacífico que desde Guadalajara, Jalisco, utilizó el método de proyección solar y un helioscopio. Salazar Ilarregui, jefe de la Comisión de Límites con Guatemala utilizó un telescopio altazimutal de 12 pulgadas de distancia focal, mientras que Joaquín Mendizabal y Tamborrel contó con un anteojo meridiano y un telescopio altazimutal de la compañía Thoughton y Simms, y un telescopio refractor de Dollond de 3 pulgadas. Por su parte, el ingeniero Jacobo Blanco, en el pueblo de San Marcos, en el estado de Guerrero, utilizó un sextante de la fábrica de Toughton Simms y un cronómetro de Parkinson \& Frodsham. Desde el Observatorio Meteorológico y Astronómico de Mazatlán, Sinaloa, el señor Quijano utilizó un anteojo de pasos de $6 \mathrm{~cm}$ de abertura, un cronómetro marino y un telescopio con movimiento paraláctico, a su vez, el señor Bonilla en Zacatecas utilizó un telescopio ecuatorial. También participaron Heraclio Jiménez y el ingeniero José A. Brambila jefe de la Comisión de Geografía y Estadística del estado de Guanajuato; Juan Madrid encargado del observatorio meteorológico de Toluca; el profesor José María Chacón, del Colegio de San Nicolás de Hidalgo, Michoacán; Manuel Pastrana desde el Observatorio provisional en Tapachula; Rodrigo Valdés, jefe de la Comisión Militar Geográfico Exploradora de los estados de Nuevo León y Tamaulipas; y Agustín Díaz de la Comisión Exploradora de Jalapa, de quienes desconocemos el equipo óptico que utilizaron. Un caso aparte fue el grupo organizado por el director del Observatorio meteorológico y astronómico del colegio católico jesuita de Puebla, el Padre Pedro Spina S.J. [29].

\subsection{La delegación francesa}

Como muestra de la importancia de la llegada de la delegación francesa al puerto de Veracruz en octubre de 1882, varios medios impresos franceses como Le Temps, Le Rapppel y el periódico mexicano Siglo XIX [30] hicieron saber del acontecimiento a sus lectores. Las notas mencionaron que la delegación integrada por De la Grye, el hidrógrafo Héraud y el encargado de fotografía Arago, habían sido recibidos por Felipe Valle, aún estudiante de la carrera de ingeniero geógrafo de la ENI. La misión gala transportó un telescopio ecuatorial de 6 pulgadas, y uno de 4 pulgadas, un péndulo eléctrico y otro de gravedad, un sismógrafo, cronómetros y brújulas para realizar estudios de magnetismo. Como una prueba de que las comisiones contaban con el respaldo de ambas naciones, Felipe Valle, cercano allegado del director Anguiano, en un acometido diplomático los acompañó hasta la capital de México en donde con el ministro de relaciones exteriores de Francia en México, el diplomático M. de Coutouly, conversaron con el Secretario de Fomento Carlos Pacheco [31]. Aprovechando la estancia, convinieron con los astrónomos mexicanos en las estrellas fundamentales para realizar las observaciones de pasos meridianos, unieron sus cronógrafos con un cable y, para reducir el error personal en el cálculo de la longitud, cada delegación intercambió señales telegráficas desde Chapultepec a Loreto y posteriormente a la inversa [32].

\section{Epílogo}

Al fin, el esperado 6 de diciembre de 1882 llegó y los astrónomos estuvieron atentos al firmamento a partir de las 9:40 am. La oportunidad de que el azar les permitiera observar el fenómeno natural los privilegiaba. A pesar de todo, lo que ese día prevaleció en el ánimo de los astrónomos fue la incertidumbre de que las condiciones climáticas fueran óptimas.

Aunque el pronóstico del meteorológico del observatorio era de cielo despejado, en la capital de la república mexicana prevaleció el cielo nuboso. A pesar de la decepción, los grupos del OAC y los de la ENI pudieron cronometrar el segundo contacto, mientras que los de Chapultepec el primero y el segundo y lograron tomar 13 placas. A los localizados en el resto del país el clima los benefició, ya que el cielo se mantuvo despejado y pudieron cronometrar los cuatro contactos y algunos, inclusive, reportaron que no observaron el ligamento negro. De la misma forma, el clima en la capital poblana no impidió que los franceses llevaran a cabo sus mediciones y lograron tomar fotografías.

Cabe mencionar que un logro fue que un par de años después De la Grye y Anguiano publicaron un aproximado de la longitud de Puebla, derivada de la diferencia de meridianos entre el observatorio de Chapultepec al localizado en el Fuerte de Loreto. Además, aprovechando el momento, las misiones científicas continuaron intercambiando señales telegráficas con otros sitios y aunque el proceso de datos fue muy lento, De la Grye publicó en 1905 la longitud del Observatoire de Paris al de Greenwich; del de Greenwich al de Washington y de ahí al de Saint Louis, Missouri. De Saint Louis, al observatorio de Tacubaya, México -sitio a donde se trasladó el observatorio en el año de 1883- y de Tacubaya al palacio de Chapultepec [32] por lo que fueron felicitados por el éxito obtenido en el diario estatal Journal officiel de la République francaise [33].

De acuerdo al compromiso científico, todos los observadores del país le enviaron los resultados al líder de la organización mexicana utilizando la nueva red telegráfica conectada al OANCH; por el mismo medio, el Observatoire de Paris reenvió al director del Observatorio Nacional los resultados de las comisiones internacionales ubicadas en Europa, Asia, África y América.

Como parte de la campaña mediática para difundir el éxito de las delegaciones nacionales y la extranjera, se publicaron los resultados en las Memorias de la Secretaría de Fomento, Colonización, Industria y Comercio (dirigidas a la burocracia) y en el Anuario del Observatorio Astronómico Nacional para 1884 (cuyos lectores eran la élite intelectual), así como en los periódicos El Siglo XIX y Monitor Republi- 


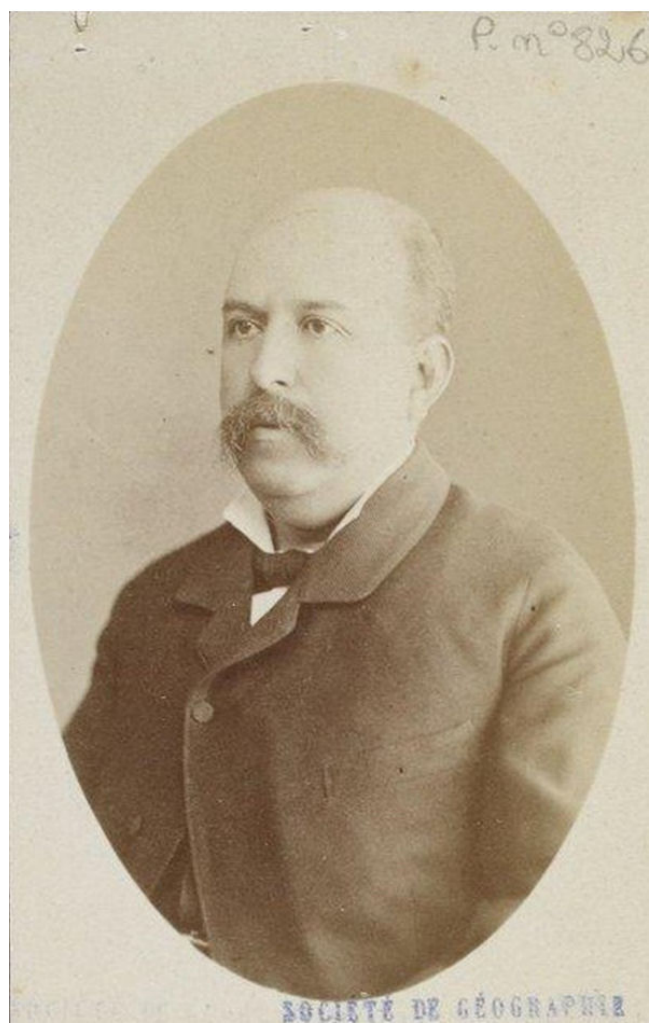

FIgURA 4. Imagen del director del Observatorio Astronómico Nacional de Chapultepec, el ingeniero civil y arquitecto Ángel Anguiano, donada a la prestigiosa Société de géographie de Paris. La fotografía se integró al catálogo internacional de ingenieros, astrónomos, o geógrafos en el año de 1883 [35].

blicano, ambos dirigidos al público en general. Extrañamente, las resoluciones de la conferencia de París y las instrucciones para cronometrar el ligamento negro se continuaron publicando durante el mes de diciembre de 1882 y enero de 1883 en El Minero Mexicano, periódico portavoz del gremio intelectual empresarial.

\section{Conclusiones}

Demostramos que la participación del ingeniero civil y arquitecto Ángel Anguiano en el evento astronómico que trascen- dió fronteras, se debió a intereses personales y no a una política científica de estado.

La colaboración le permitió legitimarse entre los integrantes de la comunidad científica internacional al demostrar capacidad organizativa y logística y posicionó al Observatorio Astronómico Nacional dentro del mundo globalizado del siglo XIX relacionado con la astronomía.

Mostramos que aprovechó sus relaciones con el poder político para gestionar el apoyo financiero para adquirir infraestructura moderna, misma que el gobierno utilizó como símbolo de progreso y no como un medio para fortalecer la ciencia nacional. Prueba de ello es que nunca hubo disponibilidad de medios para cubrir las plazas de recursos humanos. Con todo, su comprometida masa crítica intelectual fue capaz de efectuar trabajos de fotografía de primera línea, motivo por el cual (como símbolo de pertenencia al círculo hegemónico) el gobierno francés reconoció el trabajo de los observadores otorgándoles una medalla.

En otro sentido, al integrar (a través del canje) al Anuario del Observatorio Astronómico Nacional a una amplia red de publicaciones científicas editadas en los observatorios participantes en la conferencia, potencializó el intercambio de ideas entre los integrantes del OANCH y sus pares internacionales. Mientras en el espacio académico de la ENI, ascendió a profesor titular del nuevo curso Astronomía Física y Mecánica Celeste, cuya orientación teórica estaba fuertemente vinculada a los nuevos trabajos astrofotográficos del observatorio.

En resumen, la participación de Ángel Anguiano en la observación internacional del tránsito de Venus le permitió ascender como líder de la astronomía mexicana, y capitalizar las conexiones con el Observatoire de Paris, para incursionar como director del futuro Observatorio Astronómico Nacional de Tacubaya en el nuevo escenario de la astrofotografía internacional [34].

\section{Agradecimientos}

Se agradecen los comentarios de un árbitro anónimo y del doctor Alejandro Lara Sánchez, investigador del Instituto de Geofísica de la Universidad Nacional Autónoma de México, que ayudaron a enriquecer el escrito.
1. J. Galindo Trejo, El tránsito de Venus por el disco del Sol de 2012, Arqueología Mexicana 17 (2018) 49.

2. J. Galindo Trejo, y C. Allen, Maya, Observations of 13th Century Transits of Venus? Proc. Int. Astron. Union 196 (2004) 124. doi $10.1017 /$ S1743921305001328

3. G. Galilei, Noticiero Sideral, edición conmemorativa del IV centenario de la publicación de Sidereus Nuncius. Traducción del latín, a partir de la edición de Venecia 1610 (Museo Nacional de Ciencia y Tecnología, La Coruña y Madrid, 2010).

4. National aeronautics and space administration, Six millennium catalog of Venus transits: 2000 BCE to $4000 \mathrm{CE}$. Accesado el 23 de julio de 2020. https://eclipse.gsfc.nasa. gov/transit/catalog/VenusCatalog.html

5. E. A. Halley, New Method of Determining the Parallax of the Sun, or His Distance from the Earth, Abr. Trans. Roy. Soc. 6 (1809) 243; B. Schaefer, The transit of Venus and the notorious black drop effect, J. Hist. Astron. 32 (2001) 325. https : //doi.org/10.1177/002182860103200402

6. M. De la Guardia Durán, El astrónomo Ángel Anguiano: un experto aprendiz, Rev. Digit. Univ. 16 (2015) 4. 
http://www.revista.unam.mx/vol.16/num4/ art24/index.html

7. A. Gramsci, Quaderni del carcere III (Einaudi, Torino 1975) 1515.

8. S. Moreno y Kalbtk, El porfiriato. Primera etapa (1876-1901), en Historia de la Educación Pública en México, editado por F. Solana, R. Cardiel y R. Bolaños (Fondo de Cultura Económica, México, 1981), pp. 41-82.

9. Sobre la Secretaría de Fomento, Colonización, Industria y Comercio, como promotora de instituciones científicas véanse B. Martínez, y J. O. Moncada Maya, El Ministerio de Fomento, impulsor del estudio y el reconocimiento del territorio mexicano (1877-1898), Investigaciones geográficas, 74 (2011) 74 y el de L. F. Azuela, La institucionalización de las ciencias en México durante el Porfiriato, en M. L. Rodríguez Sala (coord.), Tres etapas del desarrollo de la cultura científico tecnológica en México (UNAM, México) (1996) pp. 73-84.

10. L. G. León, Los progresos de la astronomía en México desde 1810 hasta 1910, (Tipografía de la vda. de Díaz de León, México, 1911) pp. 17.

11. V. Riva Palacio, Informe que presenta al Congreso de la Unión por el Secretario de Estado y del despacho de Fomento, Colonización, Industria y Compercio de la República Mexicana Vicente Riva Palacio correspondiente al año transcurrido de diciembre de 1876 a noviembre de 1877 (Imprenta de Francisco Díaz de León, México, 1887), p. 492.

12. A. Anguiano, 'Aspecto general del cielo' Boletín del Ministerio de Fomento de la República Mexicana, 20 (1877) 1.

13. S. Zueck, Ángel Anguiano, impulsor de la biblioteca especializada del Observatorio Astronómico Nacional de Tacubaya Rev. Gen. Inf. 27 (2017) 503. DOI: https : / / doi . org/10. 5209/RGID.58215

14. A. Anguiano, Primera Memoria del Observatorio Astronómico Nacional establecido en Chapultepec comprendiendo los trabajos científicos ejecutados en él desde el día de su instalación, 5 de mayo de 1878, hasta el 31 de diciembre de 1879, presentada a la Secretaría de Fomento por el ingeniero Ángel Anguiano, director de dicho Observatorio, (Imprenta de Francisco Díaz de León, México, 1880) pp. 145-148.

15. A. Anguiano, Conferencia internacional del Paso de Venus, Anuario del Observatorio Astronómico Nacional de Chapultepec para 18822 (1881) 56.

16. Sobre la historia nipona se pueden consultar las obras: F. Bulnes, El paso de Venus en Sobre el hemisferio norte once mil leguas. Impresiones de viaje a Cuba, los Estados Unidos, el Japón, China, Cochinchina, Egipto y Europa. Estudio preliminar de José Ricardo Chaves. (Edición Europa. Edición facsimilar, México, 2012) pp. 157-165. y Moreno Corral, M. A., Odisea 1874, o el primer viaje internacional de científicos mexicanos. (Colección La ciencia para todos, México,1986). Ambos autores analizan la travesía al Japón en 1874 de los científicos liberales Agustín Barroso, Francisco Bulnes, Francisco Díaz Covarrubias, Manuel Fernández Leal y Francisco Jiménez.

17. A. Anguiano, Viaje a Europa en Comisión Astronómica, (Imprenta de Francisco Díaz de León, México, 1882) pp. 4-5.

18. P. Raposo, Observatories, instruments and practices in motion: an astronomical journey in the nineteenth-century, J. Hist. Sci. Technol. 8 (2013) 69.
19. A. Angiano, Viaje a Europa en Comisión Astronómica (Imprenta de Francisco Díaz de León, México, 1882), pp. 14-15.

20. A. Anguiano, Viaje a Europa en Comisión Astronómica, (Imprenta de Francisco Díaz de León, México,1882) pp. 16.

21. A. Anguiano, Misiones proyectadas para observar el paso de Venus de 1882, Anuario del Observatorio Astronómico Nacional de Chapultepec para el año de 18833 (1882) 113. Ministère des affaires étrangères, Conférence internationale du passage de Vénus, (Imprimerie Nationale, Paris, 1881) pp. 3233.

22. A. Anguiano, Memoria presentada al Congreso de la Unión por el Secretario de Estado y del Despacho de Fomento, Colonización, Industria y Comercio de la República Mexicana por el secretario Carlos Pacheco. Correspondiente a los años transcurridos de diciembre de 1977 a diciembre de 1882, (Tipografía de la Secretaría de Fomento, México, 1885) pp. 285; A. Anguiano, Anuario del Observatorio Astronómico Nacional de Tacubaya para el año de 18843 (1883) 97.

23. A. Angiano, Viaje a Europa en Comisión Astronómica (Imprenta de Francisco Díaz de León, México, 1882), pp. 1-9.

24. Ministère des affaires étrangères, Conference internationale $d u$ passage de Vénus (Imprimerie Nationale, Paris, 1881) 28. las instrucciones para cronometrar los contactos fueron reimpresas en A. Anguiano, Ligamento negro o gota que se forma en los Pasos de Venus y de Mercurio por el disco del Sol, Anuario del Observatorio Astronómico Nacional de Chapultepec para 1883, 3 (1882) 117.

25. A. Anguiano, Informe del director del Observatorio Astronómico Nacional de Tacubaya. Memoria presentada al Congreso de la Unión por el Secretario de Estado y del Despacho de Fomento, Colonización, Industria y Comercio de la República Mexicana. C. Pacheco, Correspondiente a los años transcurridos de diciembre de 1977 a diciembre de 1882 (Tipografía de la Secretaría de Fomento, México, 1885) pp. 283.

26. S. Zueck, Primer acto de globalización científica en la república mexicana. El tránsito de venus por enfrente del disco solar. Circulación del Conocimiento Científico en México: El Anuario del Observatorio Astronómico Nacional de Chapultepec (Primera época) (Universidad Nacional Autónoma de México, México, 2015) pp. 267.

27. A. Anguiano, Informe del director del Observatorio Astronómico de Tacubaya (documento num.4). Memoria presentada al Congreso de la Unión por el Secretario de Estado y del Despacho de Fomento, Colonización, Industria y Comercio de la República Mexicana. C. Pacheco, Correspondiente a los años transcurridos de diciembre de 1977 a diciembre de 1882 . (Tipografía de la Secretaría de Fomento, México, 1885) pp. 288; A. Anguiano, Anuario del Observatorio Astronómico Nacional para 1884 (1883) 77; A. Anguiano, "Departamentos astronómicos para la observación del paso de Venus" El Monitor Republicano 32 (1882) 2.

28. A. Anguiano, Informe del director del Observatorio Astronómico Nacional de Tacubaya (documento num 4.) Memoria presentada al Congreso de la Unión por el Secretario de Estado y del Despacho de Fomento, Colonización, Industria y Comercio de la República Mexicana. Carlos Pacheco. Correspondiente a los años transcurridos de diciembre de 1977 a diciembre de 1882 , 
(Tipografía de la Secretaría de Fomento, México, 1885) p. 281;

Anguiano, A, Observación del paso de Venus, Anuario del Ob-

servatorio Astronómico Nacional de Tacubaya para 1884, 4 (1883) 89. 
29. F. Valle, "Llegada de la comisión francesa para la observación del paso de Venus" El Siglo XIX, (1882) num. 13825, p. 3; La redacción, "La comissión francaise" Le Rappel, (1882) 4634, s/p.; La redacción, "Mexique" Le temps, (1882), 21, 7888, s/p.

30. Bouquet de la Grye, A, Mission du mexique. Mem. Acad. Sci. Inst. France, (Imprimerie Gauthier-Villars, Paris, 1905) pp. 148.

31. Bouquet de la Grye, A, Mission du Mexique. Mem. Acad. Sci. Inst. France (Imprimerie Gauthier-Villarars, Paris, 1905) pp. 211.
32. Bouquet de la Grye, A, Mesures des plaques, Puebla, Mem. Acad. Sci. Inst. (Imprimerie Gauthier-Villarars, Paris, 1905) pp. 241.

33. “Académie des sciences" Journal officiel de la République francaise, 15 (1883) 1423.

34. Valleto y Cía, Fotografía de Ángle Anguiano (México, 13 de marzo de 1883). Colección digital: fotografías en placa de vidrio de la Société de Géographie, https: / / catalogue. bnf.fr/ark:/12148/cb405861372 